\title{
Rechnung mit vielen Unbekannten
}

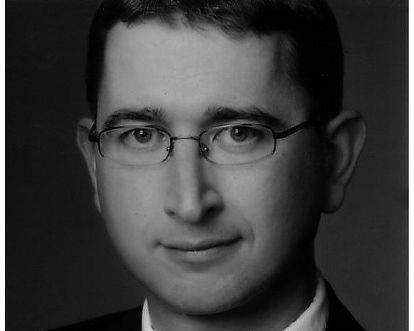

VON JOACHIM ROCK

Dr. Joachim Rock ist Politikwissenschaftler und Diplom-Verwaltungswirt und arbeitet als Grundsatz- und Europareferent für den Paritätischen Wohlfahrtsverband - Gesamtverband e. V. E-Mail joachim.rock@paritaet.org
Der Koalitionsvertrag von CDU, CSU und FDP vom Oktober 2009 soll die Vorhaben der Regierungsparteien auf Bundesebene in der laufenden Legislaturperiode auflisten. Ein Lektürebericht aus sozialwirtschaftlicher Sicht von der Arbeitsmarktpolitik bis zum Zivildienst offenbart allerdings viele Lücken.

Bei den ersten vier Bundestagswahlen nach der Wiedervereinigung erzielten die beiden mitgliederstärksten Parteien CDU/CSU und SPD bei Verschiebungen untereinander zusammen jeweils 77 Prozent. Im Gegensatz zu dieser erstaunlichen Stabilität steht das Ergebnis der zurückliegenden Bundestagswahl, in der die Parteien zusammen nur auf 56 Prozent kamen. Dies und die niedrigste Wahlbeteiligung bei einer Bundestagswahl sind Symptome eines Vertrauens- und Orientierungsverlustes der Politik. Umso gespannter durfte man sein, welche Antworten die von 139 Repräsentanten der Parteien in zehn Arbeitsgruppen entwickelte Koalitionsvereinbarung der neuen Bundesregierung auf diese Entwicklung geben.

Dass die Lektüre viele Fragen offen lässt, liegt auch an den in den 133 Seiten enthaltenen 135 Überprüfungsankündigungen, die den Eindruck erwecken, die Koalitionspartner müssten erst auf fremden Terrain eine Positionsbestimmung vornehmen. Einen roten Faden, gar eine Vision, sucht man in der Vereinbarung vergeblich.

Konkrete Ankündigungen finden sich selten, umso häufiger dagegen die Ankündigung der Berufung von Kommissionen. Allein acht Kommissionen will die Bundesregierung neu einsetzen. Mit jeder Kommission verbinden sich gleich mehrere ungelöste Fragenkomplexe. Von einer »geistig-moralischen Wende", wie sie Vizekanzler Westerwelle beschwor, findet sich nichts im Koalitionsvertrag. Das erste Wort des Koalitionsvertrages lautet Wachstum, das erste von fünf inhaltlichen Kapiteln ist der Wirtschaft gewidmet. Während Regierungen anderer Länder eine qualitativ andere Politik fordern und formulieren, ignoriert der Koalitionsvertrag Dimension, Ursachen und Folgen der Finanzkrise. Dies soll im Folgenden an den Ankündigungen in zentralen Politikfeldern belegt werden.

\section{Arbeitsmarktpolitik}

Als Verbesserungen für die Betroffenen präsentierten CDU/CSU und FDP mit dem Koalitionsvertrag zunächst eine Verbesserung der Zuverdienstmöglichkeiten für Hartz IV-Empfänger, eine Verdreifachung des "Schonvermögens « für die Alterssicherung und eine nicht näher konkretisierte Verbesserung des Schutzes selbst genutzten Wohnraums. Während bislang 100 Euro Erwerbseinkommen anrechungungsfrei waren, soll dieser Anteil erhöht und zugleich das Schonvermögen von 250 Euro auf 750 Euro pro Lebensjahr bei maximal 48.750 Euro verdreifacht werden. Beide Parteien distanzieren sich damit von früheren Forderungen, waren sie es doch, die 2004 im Vermittlungsverfahren die Absenkung des Schonvermögens von 520 Euro auf 200 Euro pro Lebensjahr ebenso unterstützt hatten wie eine Senkung des Höchstbeitrages von 33.800 Euro auf $13.000 \mathrm{Eu}$ ro.

So begrüßenswert die Maßnahmen dennoch sind, so gering ist ihre Relevanz für die Mehrheit der betroffenen Menschen. Bislang wurden nur 0,2 Prozent der Leistungsanträge wegen zu hoher Vermögensrücklagen abgelehnt. Die Koalitionspartner gehen für die Erhöhung des Schonvermögens von einem im Vergleich überschaubaren Finanzierungsvolumen von 300 Millionen Euro aus. Zur Verbesserung der Zuverdienstmöglichkeiten enthält der Koalitionsvertrag keine konkreten Aussagen.

Im Koalitionsvertrag wird das Ziel formuliert, die Brückenfunktion von sogenannten Mini- und Midi-Jobs in reguläre Beschäftigungsverhältnisse zu stärken. Im Gegensatz dazu steht jedoch die angekündigte Prüfung der Ausweitung der sozialversicherungsfreien Mini-Jobs ebenso wie die angekündigte Evaluation und mögliche Aufhebung der Mindestlohnregelungen. Beides kann dazu führen, die Ausweitung des ohnehin gewachsenen Niedriglohnsektors zusätzlich zu befördern.

Zur Zukunft der Jobcenter postuliert die Koalitionsvereinbarung die getrennte Aufgabenwahrnehmung durch Kommunen und die Bundesagentur für Arbeit. Das beträfe über 300 Arbeitsgemeinschaften, in denen die Verantwortung für Vermittlung, Maß- 


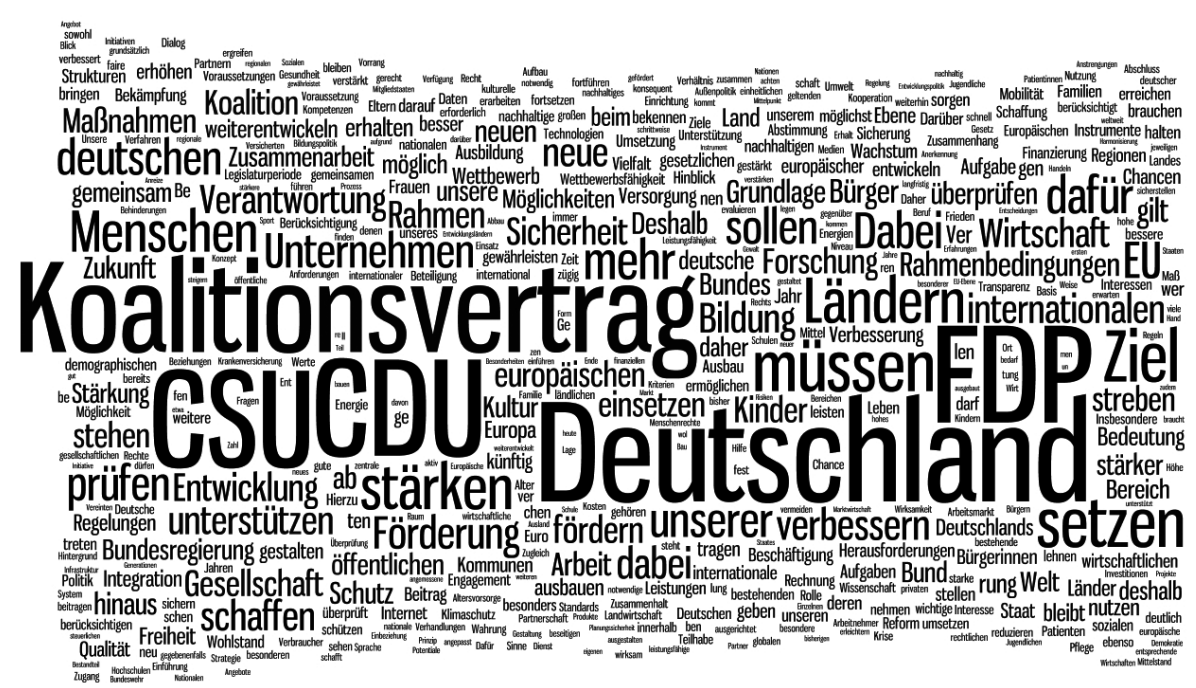

Der 133-seitige Koalitionsvertrag von CDU, CSU und FDP auf einen Blick: der Text der Vereinbarung als Grafik, in der die 500 am meisten vorkommenden Wörter - ohne Füllwörter wie Artikel - ihrer Häufigkeit entsprechend groß dargestellt werden.

nahmen und Geld einerseits und Miete und Sozialberatung andererseits getrennt würden. Lediglich die existierenden 69 Optionskommunen sollen fortbestehen können. Für die große Mehrheit der Arbeitsgemeinschaften würden damit Doppelarbeiten festgeschrieben, obwohl sich bereits in der zurückliegenden Legislaturperiode alle Ministerpräsidenten für eine Grundgesetzänderung zur Legalisierung der bestehenden Arbeitsgemeinschaften ausgesprochen hatten. $\mathrm{Zu}$ sätzlich will die Regierung größere Entscheidungsspielräume vor Ort ermöglichen, allerdings bei Reduzierung der arbeitsmarktpolitischen Instrumente.

Da für die Arbeitsförderung weniger nicht mehr, sondern schlicht weniger bedeutet, ist das angesichts der auch mit dem Auslaufen des Kurzarbeitergeldes wachsenden Probleme am Arbeitsmarkt kein Grund für Optimismus.

\section{Armutsbekämpfung}

Auf ein Bekenntnis zur Fortsetzung der Armuts- und Reichtumsberichterstattung sowie zur Fortsetzung des gemeinsamen Sozialmonitorings von Bundesregierung und Wohlfahrtsverbänden verzichten die Koalitionspartner. Es ist jedoch davon auszugehen, dass beides fortgesetzt werden wird. Für das Sozialmonitoring liegt bereits eine entsprechende Absichtserklärung des Chefs des Bundeskanzleramtes vor.

Auffällig ist die Zurückhaltung im Bereich der Regelsätze und bei der Bekämpfung der Kinderarmut. Obwohl früh absehbar war, dass das Bundesverfassungsgericht grundlegende Korrekturen bei der Bemessung der Regelsätze beschließen wird, beschränkten sich die Koalitionspartner auf die lapidare Formulierung, dass Kinderarmut »bekämpft « werden soll. Nicht einmal das Bekenntnis zu einer Ausweitung des
Kinderzuschlags hat Eingang in den Koalitionsvertrag gefunden. Angesichts der skandalösen Armutsbetroffenheit von Kindern ist das ein mehr als ernüchterndes Verhandlungsergebnis. Ganz anders bewerten die Koalitionspartner offensichtlich das Problem der zunehmenden Altersarmut, dem ein eigener Absatz mit konkreten Ankündigungen (siehe unten) gewidmet ist.

Trotz der Probleme der Pauschalierung sozialer Leistungen, wie sie sich in den zurückliegenden fünf Jahren gezeigt haben, will die Koalition eine Pauschalierung von Unterkunfts-, Energie- und Nebenkosten ebenso prüfen wie die Einführung eines Bürgergeldes, wie es durch die FDP und Teile der CDU gefordert wird.

\section{Politik für Menschen mit \\ Behinderungen}

Überwiegend positiv zu bewerten sind die Vereinbarungen im Bereich der Behindertenpolitik (vgl. auch den Beitrag von Claudia Zinke in diesem Heft). Die Koalitionspartner bekennen sich zur Herstellung von Barrierefreiheit und zu den Zielen und Inhalten der UN-Konvention über die Rechte von Menschen mit Behinderungen. Dieses Anliegen als ein Querschnittsthema taucht im Koalitionsvertrag mehrfach auf. Darüber hinaus wird die Umsetzung eines Aktionsplans zur Umsetzung der UN-Konvention angekündigt. Leider fehlt jedoch ein Bekenntnis zur inklusiven Bildung.

\section{Bildungspolitik}

Mit den auf Bundesebene begrenzten Kompetenzen im Bildungsbereich möchte die neue Regierung die private Vorsorge fördern und Neugeborenen »beispielsweise « ein Zukunftskonto mit einem Startguthaben von 150 Euro einrichten. Während angesichts der Unterrepräsentation von Studierenden aus einkommensschwachen Familien ein Ausbau der bedarfsabhängigen Förderung angezeigt wäre, setzt die neue Bundesregierung im Gegenteil auf eine einkommensunabhängige Förderung von Stipendiaten. Nach sozialen Kriterien ist das eine Fehlallokation der begrenzten Mittel in dem Bereich.

Ausdrückliche Erwähnung finden die Wohlfahrtsverbände als Partner der Regierung im Bildungskapitel, bei der Aufgabe, Bildungschancen für Ältere zu schaffen. Angesichts des Beitrags der Verbände sowohl als Dienstleistungsanbieter als auch bei der Förderung des gesellschaftlichen Engagements ist zu hoffen, dass die neue Regierung in der praktischen Arbeit in deutlich mehr Bereichen partnerschaftlich agieren wird.

\section{Bürgerschaftliches Engagement}

Dies wäre gerade beim bürgerschaftlichen Engagement zu wünschen. Die Wohlfahrtsverbände werden an dieser Stelle jedoch nicht erwähnt. Betont werden der enge Zusammenhang von Rechten und Pflichten und die Bedeutung der Vermittlung von Werten an Jugendliche.

Paradoxerweise streben die Koalitionspartner zur Förderung der Zivilgesellschaft ein Gesetz zur Förderung des bürgerschaftlichen Engagements an, mit dem u. a. Förderprogramme gebündelt, abgestimmt und weiterentwickelt werden sollen. Das gilt umso mehr, als die Koalitionspartner keine eigenen Investitionen im Engagement ankündigen, sondern lediglich ein Bekenntnis zur Förderung von Investitionen in diesem Bereich. Offenbar soll der staatliche Einfluss erheblich gesteigert werden. $\mathrm{Ob}$ und wie das geschehen soll, lässt die Koalitionsvereinbarung jedoch im Dunkeln.

\section{Europapolitik}

Grundsätzlich enthält der Koalitionsvertrag ein klares Bekenntnis zum Subsidiaritätsprinzip. Der Einfluss der CSU macht sich diesbezüglich deutlich positiv bemerkbar. Der weiter wachsende Einfluss des europäischen Wettbewerbs- und Beihilferechts wird jedoch nur an zwei Stellen konkret problematisiert: bei der Regionalförderung und dem Ziel, das Branntweinmonopol bis 2017 zu verlängern. Betont wird jedoch die nationale Verantwortung für die Sozialpolitik sowie die Ablehnung grenzüberschreitender Sozialsysteme zugunsten von hohen Standards in Deutschland.

\section{Familienpolitik}

Die Familienpolitik ist eines der zentralen Themen des Koalitionsvertrages. Die 
Bedeutung der Familien und dabei insbesondere die Verantwortung der Eltern werden mehrfach hervorgehoben. Neben diesem Grundsatz werden die Herstellung gleicher Chancen von Frauen und Männern ebenso wie die Bedeutung der Wahlfreiheit zwischen Familienleben und Erwerbstätigkeit betont. Deutlich erkennbar ist das Bemühen der Koalitionspartner, die Rahmenbedingungen der Kinderbetreuung zu verbessern, indem die Bedeutung eines quantitativen und qualitativen Ausbaus von Kinderbetreuung einschließlich der Tagespflege betont wird. Der (frühkindlichen) Bildung wird dabei ein besonderer Stellenwert eingeräumt.

Über den Ausbau von Infrastrukturangeboten zur Verbesserung der Vereinbarkeit von Familie und Beruf hinaus, bekennen sich die Koalitionspartner zu einer stärkeren finanziellen Förderung von Familien. Neben der Anhebung des Kinderfreibetrages zum 1. Januar 2010 auf $7.008 \mathrm{Eu}-$ ro und der Anhebung des Kindergeldes sollen dazu weitere steuerliche Entlastungen im Umfang von bis zu 24 Milliarden Euro im Jahr erreicht werden. Leistungen nach dem Unterhaltsvorschussgesetz sollen ausgebaut und zusätzlich ein Betreuungsgeld von 150 Euro monatlich ab 2013 eingeführt werden. In welcher Form diese familienpolitische Leistung gewährt werden soll, bleibt offen, so dass über mögliche Fehlsteuerungen bislang nur spekuliert werden kann. Festzuhalten ist jedoch, dass dadurch zumindest zum Teil Finanzierungslücken geschlossen werden, die durch die Ablösung des Erziehungsgeldes durch das Elterngeld entstanden sind. Unter diesem Aspekt ist dieses Instrument differenziert zu betrachten.

Dass Kinderlärm nicht mehr gerichtlich sanktioniert werden soll, ist erklärtes Ziel der Koalition. Positive Akzente setzt die Koalition auch im Bereich des Kinderschutzes, indem der Ausbau präventiver Maßnahmen und früher Hilfen als Ziele formuliert werden. Die in der vergangenen Legislaturperiode gescheiterte Verabschiedung eines Kinderschutzgesetzes soll in der neuen Legislaturperiode nachgeholt werden. Die Koalitionspartner verständigten sich zudem auf die Förderung von Hilfen zur Bekämpfung von Gewalt gegen Frauen und wollen einen Ausbau der Hilfeinfrastruktur fördern, soweit dies im Rahmen der Bundeskompetenzen liegt.

\section{Gesundheitspolitik}

Der bestehende Leistungskatalog der Krankenversicherung wird als »Basis « für die eigene Gestaltung des Krankenversicherungsschutzes angesehen. Darüber hinausgehende Angebote sollen gefördert und Anreize für ein kostenbewusstes Verhalten geschaffen werden. Die Arbeitgeberbeiträge zur Gesetzlichen Krankenversicherung sollen festgeschrieben und langfristig ein einkommensunabhängiger Arbeitnehmerbeitrag mit einem sozialen Ausgleich für Geringverdiener eingeführt werden. Die Ausgestaltung einer solchen Kopfpauschale bleibt offen und soll durch eine Kommission geklärt werden. Wegen der zahlreichen administrativen Probleme und der erheblichen Kosten einer Umstellung ist damit in dieser Legislaturperiode nicht zu rechnen.

Anders verhält es sich mit der angekündigten Vereinfachung und Reduzierung des morbiditätsorientierten Risikostrukturausgleichs (RSA), der ein wesentliches Instrument ist, um gerade Menschen mit chronischen Erkrankungen Aussichten auf eine bedarfsgerechte Versorgung zu verschaffen. Einschnitte im RSA hätten zur Folge, dass Kassen ihre Leistungsangebote gerade für die besonders Betroffenen einschränken müssten. Nach dem Fortschritt der lang erkämpften Einführung des Morbi-RSA wäre das ein deutlicher Rückschritt für die Versorgung.

Verbessert werden soll die Möglichkeit, neben Leistungen der Gesetzlichen Kranken- und Pflegeversicherung entsprechende Dienstleistungen anzubieten. Dies mache eine Verbesserung der wettbewerblichen Strukturen notwendig. Nicht ersichtlich ist, was damit gemeint ist, ist doch gerade das Pflegeversicherungsgesetz als » Marktschaffungsgesetz « konzipiert worden.

\section{Migrations- und Integrationspolitik}

Im Bereich der Migrationspolitik werden die unterschiedlichen Positionen der Regierungsparteien besonders deutlich. Dies drückt sich abermals in einer Vielzahl von Evaluierungsvorhaben und Prüfaufträgen aus, beispielsweise bei den Erfahrungen mit der Optionsregelung, das Sachleistungsprinzip nach dem Asylbewerberleistungsgesetz sowie die Praxis der Abschiebehaft. Deutlich wird aber auch, dass bei der Förderung von Spracherwerb und Integration allgemein noch stärker als in der Vergangenheit auf Verpflichtungen gesetzt wird.

\section{Pflege}

Die Notwendigkeit einer Neudefinition von Pflegebedürftigkeit wird betont. Besondere Betreuungsbedarfe sollen dabei berücksichtigt werden, ohne dass jedoch explizit auf die im Mai 2009 vorgelegten Ergebnisse des Regierungsbeirates zur Überprüfung des Pflegebedürftigkeitsbegriffs Bezug genommen wird.

Die notwendige Entbürokratisierung im Bereich der Pflege wird von den Koalitions- partnern an mehreren Stellen betont. $\mathrm{Zu}$ Recht stellen sie das Ziel, der Pflege am Menschen deutlich mehr Zeit zu ermöglichen, in den Vordergrund. Bei den Qualitätsprüfungen soll auch aus diesem Grund die Ergebnisqualität Vorrang vor der Strukturqualität erhalten. Maßnahmen zur Vereinbarkeit von Pflege und Beruf sollen verbessert und die Attraktivität des Pflegeberufs verbessert werden.

Die Pflegeversicherung soll als » Element der sozialen Sicherung « erhalten bleiben. Neben die bestehende Pflegeversicherung soll eine obligatorische private kapitalgedeckte Pflegesicherung treten. Auf die wiederum von einer Kommission zu erarbeitende Konkretisierung darf man gespannt sein. In jedem Fall wird die weitere Abkehr von der paritätischen Finanzierung damit fortgeschrieben, ohne dass angesichts der langen Anlaufphase für die Bildung eines Kapitalstocks sowie des Verhältnisses von Zinsen und Inflation kurz- und mittelfristig mehr Geld für die Pflege zur Verfügung stünde.

\section{Renten- und Alterssicherungspolitik}

In der Renten- und Alterssicherungspolitik strebt die neue Bundesregierung eine Ausweitung der Leistungen gegenüber dem Status quo an. So soll die Rentenangleichung zwischen Ost und West noch in dieser Legislaturperiode realisiert werden. Darüber hinaus wird geprüft, die Anrechnung von Erziehungszeiten zu verbessern. Die kapitalgedeckte Altersvorsorge soll gestärkt und Selbständigen der Zugang ermöglicht werden. Darüber hinaus sollen langjährig Versicherte in der Rentenversicherung armutsfeste Renten erhalten, was auf eine Wiedereinführung der 1992 abgeschafften Rente nach Mindesteinkommen hindeuten könnte. Details bleiben allerdings auch hier einer Kommission vorbehalten.

Die seinerzeit durch die SPD durchgesetzte »Ausstiegsklausel « bei der Rente mit 67 sieht vor, dass 2010 - zwei Jahre vor Beginn der Anhebung des Renteneintrittalters- geprüft wird, ob die Beschäftigungsmöglichkeiten für Ältere eine Einführung der Rente mit 67 überhaupt zulassen. Dies ist nach Meinung von Gewerkschaften und Wohlfahrtsverbänden nicht der Fall. Der Koalitionsvertrag enthält dazu nichts.

\section{Zivildienst}

Zum 1. Januar 2011 sollen Wehr- und Zivildienst auf sechs Monate reduziert werden. Darüber hinaus soll die abschnittsweise Ableistung des Zivildienstes geprüft werden. Unter diesen Umständen wird die Bedeutung des Zivildienstes künftig deut- 
lich sinken. Ein massiver Ausbau des freiwilligen sozialen Jahres und die Möglichkeit einer freiwilligen Verlängerung des $\mathrm{Zi}$ vildienstes sollten deshalb mindestens als Ausgleich realisiert werden.

\section{Steuer- und vergaberechtliche Vorhaben}

Ausdrücklichen Handlungsbedarf sieht die Bundesregierung im Bereich der Umsatzsteuerermäßigung. Eine Kommission soll eine Umstellung auf die Ist-Besteuerung ebenso prüfen wie den Katalog der ermäßigten Mehrwertsteuersätze. Vorab haben die Koalitionspartner bereits die Anwendung des ermäßigten Umsatzsteuersatzes auf die etwa 45.000 Beherbergungsbetriebe beschlossen. Dies führt zu Mindereinnahmen von etwa einer Milliarde Euro.

Die Regierung betont das Ziel, insbesondere bei der Umsatzsteuer Wettbewerbsgleichheit zwischen kommunalen und privaten Anbietern herzustellen. Leistungen der Daseinsvorsorge sollen dadurch jedoch ausdrücklich nicht belastet werden.

Die Stärkung des Wettbewerbs ist auch das Ziel der bereits begonnen Reform des Vergaberechts. Diese soll bis Ende 2010 abgeschlossen sein. Dabei sollen die Ergebnisse der Erhöhung der Schwellenwerte in Vergabe- und Vertragsordnung für Bauleistungen (VOB) und der Verdingungsordnung für Leistungen (VOL) überprüft und mehr Transparenz im Unterschwellenbereich geschafft werden.

Bei den Basel II-Regelungen und den Vorschriften zur Rechnungslegung streben die Koalitionspartner Lockerungen an, um in Krisenzeiten prozyklische Entwicklungen abzumildern.

\section{Das Sichere ist nicht sicher ...}

Wenig exponiert, auf Seite 21, findet sich der Satz, dass alle Maßnahmen des Koalitionsvertrages unter Finanzierungsvorbehalt stehen. Die Zahl der von diesem nicht wiederholten Begriff des Finanzierungsvorbehaltes betroffenen Maßnahmen ist erheblich und reicht von dem angekündigten Entlastungsvolumen einer Steuerreform von 24 Milliarden Euro im Jahr bis zur Einführung einer pauschalen Gesundheitsprämie, deren zusätzliche Kosten selbst in der CDU/CSU auf bis zu 26 Milliarden Euro geschätzt werden.

Darüber hinaus will die Koalition krisenbedingte Beitragsausfälle in den Sozialversicherungen aus Steuermitteln ausgleichen. Das betrifft u. a. die Bundesagentur für Arbeit. Während diese 2009 keine separaten Bundeszuschüsse erhielt, sind 2010 mindestens 16 Milliarden Euro notwendig. In der Krankenversicherung werden sich die staatlichen Zuschüsse von 7,2 Milliarden 2009 in 2010 auf voraussichtlich 15,7 Milliarden Euro mehr als verdoppeln. Weitere Steuerzuschüsse zum Ausgleich von Einnahmeausfällen werden auch in der Gesetzlichen Rentenversicherung notwendig werden, die bereits 2009 mit 80 Milliarden Euro aus Steuermitteln bezuschusst wurde. Hinzu kommen die noch von der Vorgängerregierung beschlossenen steuerlichen Entlastungen, die zum 1. Januar 2010 in Kraft traten. Sie haben ein Volumen von 21 Milliarden Euro.

Geplant ist eine Bruttokreditaufnahme von 343 Milliarden Euro, die die Schulden des Bundes in 2010 auf über eine Billion Euro erhöhen werden. Allein die jährlichen Zinszahlungen machen etwa 40 Milliarden Euro aus. Einer höheren Verschuldung sind durch die Schuldenbremse im Grundgesetz Grenzen gesetzt. Etwa zehn Milliarden Euro sind deshalb ab 2011 jährlich einzusparen.

Städte und Kommunen müssen in dieser Legislaturperiode bereits nach jetzigem Stand 50 Milliarden Euro neue Schulden aufnehmen. Die Realisierung des Rechtsanspruchs auf einen Betreuungsplatz ab 2013 ist deshalb und wegen des Fachkräftemangels unsicher.

Die Absicht, die Sozialversicherungsbeiträge in der Legislaturperiode dauerhaft unter 40 Prozent halten zu wollen, wird sich bei dem bestehenden Niveau von 39,55 Prozent und angesichts der wachsenden finanziellen Herausforderungen nur bei erheblichen Einschnitten bei den Leistungen einlösen lassen. Entsprechende Ankündigungen sind nach der Landtagswahl in Nordrhein-Westfalen im Mai 2010 zu erwarten.

Völlig offen ist, wie die Formelkompromisse zwischen den Koalitionspartnern ausgelegt werden. Ein Vertrag setzt juristisch übereinstimmende Willenserklärungen mindestens zweier Parteien voraus. Betrachtet man die Koalitionsvereinbarung der neuen Bundesregierung, so kann davon keine Rede sein. Insbesondere CSU und FDP präsentieren sich in allen wesentlichen Fragen als uneinig. Als promovierte Physikerin ist die Bundeskanzlerin gewohnt, mit Unbekannten und Variablen zu rechnen. Diese Kompetenz wird sie in der laufenden Legislaturperiode brauchen können.

\section{Wie verbindlich sind eigentlich Koalitionsverträge?}

"Rechtsnatur und Verbindlichkeit von Koalitionsverträgen sind umstritten. Teils werden sie als Verfassungsverträge angesehen, teils als politisch - nicht aber rechtlich - bindende Absprachen qualifiziert, als 'politische Geschäftsgrundlage ‘ für die Bildung und Arbeit der Regierung. Weil Koalitionsverträge auf die Umsetzung politischer Ziele durch die Staatsorgane gerichtet sind, können sie nicht als privatrechtliche Abreden angesehen werden. Gegen ihre Einordnung als verwaltungsrechtliche Verträge wird vorgebracht, bei ihnen gehe es weder um den Vollzug öffentlich-rechtlicher Normen noch um die Verpflichtung zum Erlass eines Verwaltungsaktes. Es handele sich vielmehr um Gegenstände des Verfassungsrechts. Einigkeit besteht, dass Koalitionsverträge gerichtlich nicht einklagbar und vollstreckbar sind. Ihre faktische Wirkungskraft entfalten sie aus politischen Erwägungen. Kein Koalitionspartner wird sich ohne Not von den getroffenen Absprachen lösen und dem Verdacht der politischen Unzuverlässigkeit aussetzen. Dem Koalitionsvertrag widersprechendes Verhalten eines Koalitionspartners könnte von dem anderen Partner in aller Öffentlichkeit als Bruch eines verfassungsrechtlichen Vertrages dargestellt werden. Sanktioniert wird die Vereinbarung durch die Hoffnung, dass sich die wechselseitige Verknüpfung von Ansprüchen und Pflichten für die eigene Partei vorteilhaft auswirkt. Abredewidriges Verhalten kann zu Ansehensverlust oder gar zu dem Entzug der Regierungsmacht führen.«

Aus einer Expertise des Wissenschaftlichen Dienstes des Deutschen Bundestags vom 26. Oktober 2009 Quelle: http://www.bundestag.de/dokumente/analysen/ 2009/Koalitionsverhandlungen_-_Koalitionsvertrag.pdf 